\title{
Implementation of Watershed Segmentation
}

\author{
Lalitha $\mathbf{K}^{1}$, Amrutha $\mathbf{R}^{1}$, Stafford Michahial ${ }^{1}$, Dr M Shivakumar ${ }^{2}$ \\ Asst Professor, Dept of IT, GSSSIETW ${ }^{1}$ \\ Prof \& Head, Dept. of EIE, GSSSIETW ${ }^{2}$
}

\begin{abstract}
This paper focuses on marker based watershed segmentation algorithms. As marker based watershed segmentation algorithm causes over segmentation and cause noise in the image produced. So to reduce these problem different researchers has proposed different solutions, but the best solution is to use bilateral filter. The main objective of this paper is to find the gaps in existing literature. The different segmentation techniques are reviewed and found that marker based is best in most of cases because it marks the regions then segment them. But optimizing the marking regions is still an area of research.
\end{abstract}

Keywords: External markers, Gradient, Internal markers, Image segmentation, Watershed.

\section{INTRODUCTION}

The image segmentation is significance problem in different fields of computer vision and image processing. Image segmentation is the process of partitioning a digital image into multiple segments knows as set of pixels. The goal of segmentation is to simplify change the representation of an image into something that is more meaningful and easier to analyze. Image segmentation is typically used to locate objects and boundaries (lines, curves, etc.) in images. It is the process of assigning a label to every pixel in an image such that pixels with the same label share certain visual characteristics.

The result of image segmentation is a set of segments that collectively cover the entire image, or a set of contours extracted from the image. Each of the pixels in a region are similar with respect to some characteristic or computed property such as color, intensity, or texture. The goal of segmentation operation is to simplify the image without discarding important image features.

Image segmentation process in three stages. The first is image pre-processing, then input image is converted into gradient image further it is applied by watershed transform,object discrimination, where objects are grossly separated into groups with similar attributes. Third stage is object boundary clean up, where object boundaries are reduced to single-pixel widths. In recent years several popular methods have been developed for image segmentation.[1].

\section{A. Thresholding method}

The simplest method of image segmentation is called the thresholding method. This method is based on a threshold value to turn a gray-scale image into a binary image.

\section{B. Split and merge method}

This method is used for the division of the image. If it is found non-uniform (not homogeneous), then it is split into four son-squares (the splitting process).

\section{Histogram based method}

Histogram based methods are very efficient when compared to other image segmentation methods because they typically require only one pass through the pixels. In this technique, a histogram is computed from all of the pixels in the image, and the peaks and valleys in the histogram are used to locate the clusters in the image.

\section{Edge detection}

The first region-growing method was the seeded region growing method. This method takes a set of seeds as input along with the image. The seeds mark each of the objects to be segmented. The regions are iteratively grown by comparing all unallocated neighbouring pixels to the regions.

\section{E. Watershed transformation:}

The watershed transform has interesting properties that make it useful for much different image segmentation application. In image segmentation the objects in the image are separated and labelled In image segmentation the objects in the image are separated and labelled for further analysis. This is usually done with the help of a wide range of image segmentation techniques. Each technique has its own advantages and disadvantages. The effectiveness of a particular image segmentation algorithm is determined with respect to a particular class of images. Generally a combination of two or more techniques is used to get the desired output for a particular application. The scale of segmentation is application specific and even the objects that are to be segmented. It is important to note that:

1. There is no universally applicable segmentation technique that is guaranteed to work on all images.[2]

2. No segmentation technique is perfect.

The goal however is to come up with an ad-hoc technique for segmentation of high resolution aerial imagery which is a step further in image analysis. In recent years various 
Vol. 5, Issue 12, December 2016

techniques have been developed for image segmentation. One of them is watershed technique applied on images but it has various drawbacks that is over segmentation and sensitivity to noise so to overcome this we will use marker based watershed technique to overcome these problems. This technique "mark," foreground objects and background locations. The results obtained by the segmentation of the image are generally very subjective, as it depends on the information content of the image itself. The parameters to be considered for the evaluation of these results vary from image to image.

\section{Markerbased watershed transform}

The advantages of the watershed transformation are that it is simple, instinctive knowledge, and can be parallelized. The main drawback of this method is the oversegmentation due to the presence of many local minima. To decrease the effect of severe over-segmentation, marker-controlled watershed transformations have been proposed. These are robust and flexible methods for segmenting objects with closed contours. The internal marker and external marker are initially defined. The boundaries, even if not clearly defined, are expressed as ridges between two markers and located. In markercontrolled watershed method to segment the image the external marker is obtained manually by drawing a circle enclosing object of our interest. The internal marker is determined automatically by combining techniques including Canny edge detection, thresholding and morphological operation. Modify the segmentation function so that it only has minima at the foreground and background markers. The Watershed Transform effectively combines elements from both the discontinuity and similarity based methods. Since its original development with grey-scale images, the Watershed Transform has been extended to a computationally efficient form (using FIFO queues) and applied to colour images. The main advantages of the Watershed method over other previously developed segmentation methods are[3]

A. The resulting boundaries form closed and connected regions. Traditional edge based techniques most often form disconnected boundaries that need postprocessing to produce closed regions.

B. The boundaries of the resulting regions always correspond to contours which appear in the image obvious contours of objects.[9]

\section{PROPOSED METHODOLOGY}

This paper has presented an literature survey on marker based watershed segmentation algorithms. The main objective of this paper is to find the gaps in existing literature. The different segmentation techniques are reviewed and found that marker based is best in most of cases because it marks the regions then segment them. But optimizing the marking regions is still an area of research. However it has found that most of existing techniques uses the bilateral filter. But as it is known in prior bilateral filter is unable to reduce salt and pepper noise. So in near future to reduce this problem we will integrate marker based watershed segmentation algorithm with hybrid median filter to improve the performance. The main drawback of this method is the over-segmentation due to the presence of many local minima. To decrease the effect of severe over-segmentation, marker-controlled by the watershed transformations have been proposed. These are robust and flexible methods for segmenting objects with closed contours. The internal marker and external marker are initially defined. The [5] boundaries, even if not clearly defined, are expressed as ridges between marker and locate. In marker-controlled watershed method to segment the image the external marker is obtained manually by drawing a circle enclosing object of our interest. A gray level co-occurrence matrix. Then, both gradient images are fused to give the final gradient image. After the initial results of segmentation, we use the merging region technique to remove small regions. It is well known that the main problem of this method is that the images we consider are often noisy, which implies that we have a lot of local minima and this leads to an over segmentation. We propose in this paper, a new method for decreasing the over segmentation of standard watershed based techniques. Our method is based on the topological gradient approach. The topological gradient has here the interesting property to give more weight to the main edges, it provides a more global analysis of the image than the Euclidean gradient or the image[10]. to noise as we show it in the numerical applications section. The gradient image is often used in the watershed transformation, because the main criterion of the segmentation is the homogeneity of the grey values of the objects present in the image. But, when other criteria are relevant, other functions can be used. In particular, when the segmentation is based on the shape of the objects, the distance function is very helpful.[6].

\section{RESULTS AND DISCUSSIONS}

We consider in this section the problem of denoising of an image and preserving features suchas edges. According to the previous section, thetopological asymptotic analysis provides the location of the edges as they are precisely defined as the most negative points of the topological gradient,the results obtained by the topological gradient algorithm. The image processed given by a $256 \times 256$ gray level image and represents some rice grains. Fig. $1 \mathrm{~b}$ is obtained by adding to the original image a Gaussian noise $(\square \square=20$ ). The reconstructed image is shown in Fig. 1c: the topological gradient method for the restoration process was applied with $\mathrm{c} 0=1$. Finally, we give in, the edges of the reconstructed image. To obtain the restored image, the topological gradient algorithm requires only 3 system resolutions for calculating $\mathrm{u} 0, \mathrm{v} 0$ and the restored image u 1 given respectively by Eqs. 4, 6, and 2. For a better edge preservation, one has to threshold the topological gradient with a small enough coefficient. In the other case, if the 
Vol. 5, Issue 12, December 2016

thresholding coefficient is set to a large value, then the edges obtained will be thick, leading to an over smoothing and a loss of an important edge information and then a degradation of the restored image. Finally, to speed up the computations, a spectral method based on the discrete cosine transform has been used for the resolution of the direct and adjoint problems. Since the coefficient $\mathrm{c}$ is equal to a constant $\mathrm{c} 0$ except on edges, then the discrete cosine transform is a good preconditioner for the conjugate gradient method. The complexity of the restoration algorithm is $\mathrm{O}(\mathrm{N} \log \mathrm{N})$ where $\mathrm{N}$ is the number of pixels of the image. Some comparisons about the computationtimes with other classical methods are presented in Jaafar Belaid et al. (2008).

\section{Segmentation using a classical watershed}

The goal of this section is to present numerical tests for the segmentation problem using mathematical morphology tools. The approach used in this work is based on the watershed transform. One should remark that we can either define the watershed of the function $u$ or of its gradient: the difference between the two definitions is that in the first case we obtain the influence zones of the processed image, while the second case gives the image edges. In both cases, the watershed gives an oversegmentation and to avoid this drawback, a markers technique can beused. We propose in this section to give numerical results based on this classical method according to the work of Beucher (1990), in which the author has proposed to use both influence zones and minima ofthe filtered image as marker criteria. Fig. 2 illustrates this approach. We have considered the same original image as previously shows the watershed of the image shows the watershed of its gradient. The over segmentation is clearly seen. We mention here, that the numerical tests given by give a segmented image with 1905 regions for a computational time of $550 \mathrm{~s}$. This over segmentation can be in a first step corrected by applying a morphological filter shows the watershed of the filtered image: the number of regions segmented is attenuated (868 regions) for a computational time of $775 \mathrm{~s}$, but the segmentation [7] result remains unacceptable.

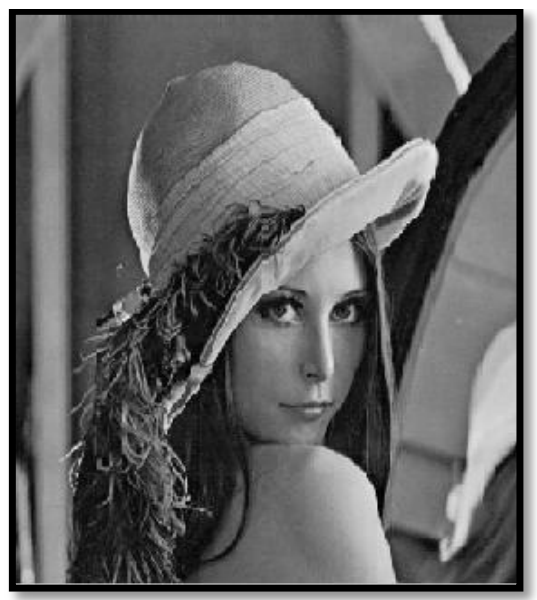

Fig. 2: Original image
However, as over segmentation is due to the fact that we obtain a lot of minima, and the use of morphological filters can only suppress some of them, then another way to act on these minima is to apply the swamping approach, by imposing markers for new minima.

The original image is the input image here we are using the lena image as the input image. Then the further preprocessed by using gradient magnitude as the segmentation function. The watershed transform cannot be applied directly to the binary image hence it is converted into RGB for better pixel response.

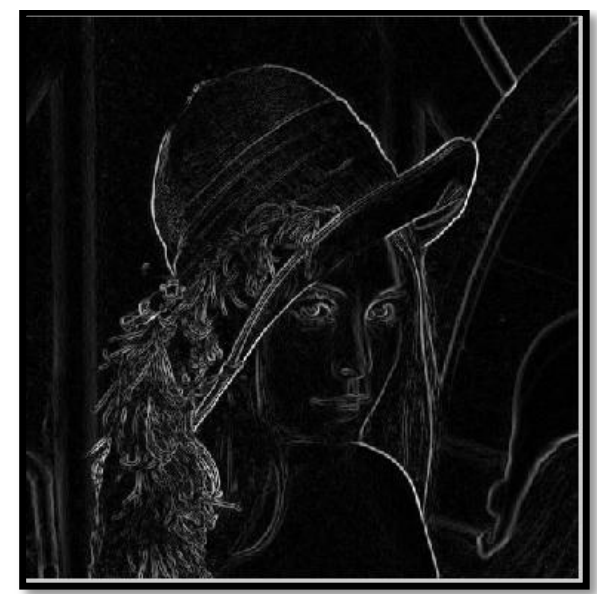

Fig3:Gradient magnitude of the image

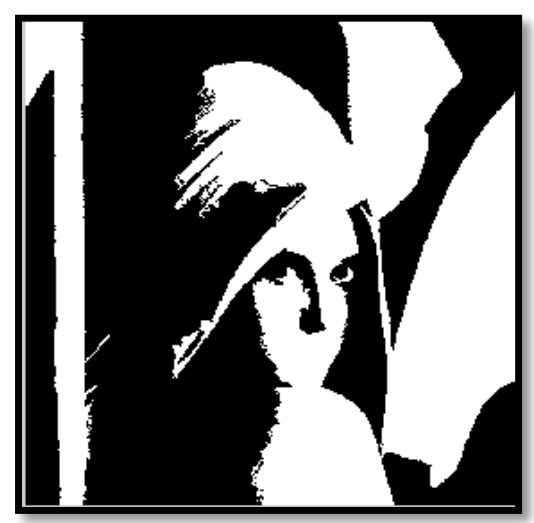

Fig.4 Thresholder image

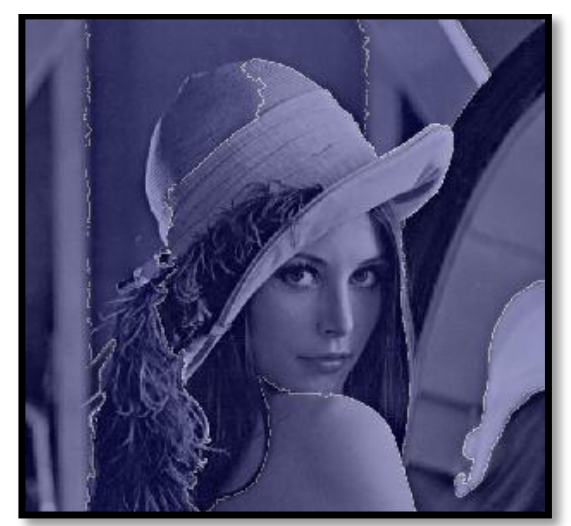

Fig.5 .Segmented image 


\section{CONCLUSION}

This paper has presented an literature survey on marker based watershed segmentation algorithms. The main objective of this paper is to find the gaps in existing literature. The different segmentation techniques are reviewed and found that marker based is best in most of cases because it marks the regions then segment them. But optimizing the marking regions is still an area of research. However it has found that most of existing techniques uses the bilateral filter. But as it is known in prior bilateral filter is unable to reduce salt and pepper noise. So in near future to reduce this problem we will integrate marker based watershed segmentation algorithm with hybrid median filter to improve the performance of proposed algorithm.[11]

\section{REFERENCES}

[1] Baojingji,jianping Lv and CaiXia Zhoa, "improved watershed algorithm based on segmentation "Xian institute of posts and telecommunications pp.103-107.

[2] Boren Li and Mao Pan, "An Improved Segmentation of High Spatial Resolution Remote Sensing Image using Marker-based Watershed Algorithm, ${ }^{\text {ece }}$ pp. 98-104, IEEE,2012.

[3] Chen Wei-bin and Wenzhou Zhejiang, "A New Watershed Algorithm for Cellular Image Segmentation Based on Mathematical Morphology," International Conference on Machine Vision and Human-machine Interface, vol.53, pp.2405-2414, IEEE, 2010.

[4] GuiMei Zhang and Ming-Ming Zhou, "Labelling watershed algorithm based on Morphological Reconstruction in Color Space," pp.51-55, IEEE, 2011.

[5] Jun Tang, "A Color Image Segmentation algorithm Based on Region Growing," vol.6, pp.634-637, IEEE, 2010.

[6] Li Cheng, Li Yan and Fan Yan Shangchun, "CCD infrared image segmentation using watershed algorithm," Third International Conference on Measuring Technology and Mechatronics Automation, vol.1, pp.680-683, IEEE,2011.

[7] Shiping Zhu, Xi Xia and Qingrong Zhang, "An Image Segmentation Algorithm in Image Processing Based on Threshold Segmentation," Third International IEEE Conference on SignalImage Technologies and internet based system, IEEE, 2008.

[8] Quan Longzhe, "Automatic Segmentation method of Touching Corn Kernels in Digital Image Based on Improved Watershed Algorithm," pp.34-37, IEEE, 2011.

[9] Wei Zhang, "The Marker-Based Watershed Segmentation Algorithm of Ore Image,” pp.472-474, IEEE, 2011.

[10] Xiaoyan Zhang, Lichao Chen, Lihu Pan and Lizhi Xiong, "Study on the Image Segmentation Based On ICA and Watershed Algorithm," Fifth International Conference on Intelligent Computation Technology and Automation, pp. 978-912, IEEE 2012 . 